Int. J. Morphol.,

33(1):119-129, 2015.

\title{
Apoptosis Durante el Desarrollo Embrionario de la Retina de Tortuga (Trachemys scripta elegans)
}

\author{
Apoptosis in Turtle Embryonic Retina (Trachemys scripta elegans)
}

Segovia, Y.

SEGOVIA, Y. Apoptosis durante el desarrollo embrionario de la retina de tortuga (Trachemys scripta elegans). Int. J. Morphol., 33(1):119-129, 2015.

RESUMEN: La apoptosis o muerte celular programada es un proceso que ocurre durante el desarrollo del sistema nervioso. El objetivo de este estudio fue observar los patrones de apoptosis que se producen durante el desarrollo embrionario de la retina, desde el estadio S16 al momento del nacimiento, mediante miscoscopía óptica y electrónica. Se utilizaron retinas de embriones de tortuga. Nuestros datos muestran que los primeros signos de apoptosis comienzan en el estadio S16, en la capa nuclear interna y alcanzan su máxima densidad tanto en la capa nuclear interna como en la capa de células ganglionares en S20, para extinguirse, prácticamente, en el momento del nacimiento. Por otra parte, la apoptosis sigue un gradiente centro-periferia.

PALABRAS CLAVE: Trachemys scripta elegans; Apoptosis; Retina; Tortuga.

\section{INTRODUCCIÓN}

La apoptosis es un mecanismo de suicidio celular que capacita a los organismos pluricelulares para controlar el número de células en los tejidos y para eliminar células individuales que ponen en peligro la supervivencia del organismo. Durante el desarrollo del sistema nervioso de vertebrados se produce un exceso de células que inmediatamente es eliminado por muerte celular para un correcto funcionamiento del organismo (Oppenheim, 1991). Este proceso de muerte celular, es crucial en la plasticidad del sistema nervioso porque ajusta el número de células nerviosas y modula la arquitectura de las conexiones neuronales.

Las primeras observaciones que se realizaron del fenómeno de la muerte celular programada fueron hechas en el contexto del desarrollo (Glücksmann, 1951). Se han determinado algunos objetivos que cumple este fenómeno durante el desarrollo de un organismo, todos encaminados en último lugar, a la eliminación de células sin utilidad en ese momento y localización: escultura de distintas estructuras, eliminación de las mismas, control de la sobreproducción de células, control de las células defectuosas y formación de células diferenciadas especiales. Y ya que el papel fisiológico de la apoptosis es crucial, la aberración de este proceso contribuye a desencadenar proce- sos patológicos, desde el cáncer a enfermedades autoinmunes y degenerativas.

Diversos estudios han demostrado que la retina de vertebrados sufre el proceso de muerte celular en diferentes estadios durante el desarrollo embrionario y es, precisamente, en estadios relativamente tardíos del desarrollo, donde se pone de manifiesto la apoptosis de muchas células diferenciadas (Young, 1984; Marín-Teva et al., 1999; Cusato et al., 2001), a la vez que se produce la sinaptogénesis (Young; Marín-Teva et al.).

El objetivo de nuestro estudio ha sido observar los procesos de apoptosis que se producen durante el desarrollo embrionario de la retina de tortuga en la capa nuclear interna (INL), en la capa nuclear externa (ONL) y en la capa de células ganglionares (GCL).

La retina de tortuga, como todas las retinas de vertebrados se organizan de acuerdo al siguiente plan básico: dos capas sinápticas (la capa plexiforme interna o proximal y la capa plexiforme externa o distal) que se hallan intercaladas entre tres capas celulares (externa, interna y capa de células ganglionares). 


\section{MATERIAL Y MÉTODO}

Animal de estudio. Este estudio ha sido realizado en embriones de la Tortuga de Orejas Rojas, o Galápago de Florida (Trachemys scripta elegans), un quelonio, que se encuentran en casi todos los lugares templados y cálidos del mundo, desde el estadio S16 al estadio S26, momento del nacimiento.

Para determinar los estadios se siguieron los criterios descritos por Yntema (1968), quién estudió el desarrollo de la tortuga Chelydra serpentina serpentina, y describió una serie de 26 estadios, basándose en las estructuras craneales y cervicales, en las extremidades y en el caparazón.

Procedimiento general para la obtención de las retinas. Todos los procedimientos realizados con los animales cumplieron la actual legislación española (Real Decreto 53/2013 de 8 de febrero, sobre protección de los animales para experimentación y otros fines científicos).

Para la obtención de las retinas se procedió a la decapitación de los animales y enucleación del globo ocular en absoluta oscuridad. Los ojos fueron hemiseccionados y la porción posterior del ojo se sumergió inmediatamente en el fijador en una solución de paraformaldehído frío al $4 \%$ en 0,1 M de tampón fosfato (PB), a pH 7,4 durante 2 horas a $4{ }^{\circ} \mathrm{C}$, para el material destinado a inmunocitoquímica. Tras la fijación, se lavaron varias veces en PB, y, se procedió a la crioprotección de las retinas en sacarosa al $15 \%$ durante 30 minutos, al 20\% durante 20 minutos y al $30 \%$ durante toda la noche a $4{ }^{\circ} \mathrm{C}$.

Las retinas dirigidas a estudios ultraestructurales convencionales se fijaron durante toda la noche en una solución de paraformaldehído al 1\%, glutaraldehído al 1,6\%, y cloruro de calcio 0,15 mM en PB 0,1 M. Tras la fijación, las retinas fueron aisladas y lavadas posteriormente varias veces en PB 0,1 M, sacarosa al 5\% y cloruro de calcio 0,15 $\mathrm{mM}$. A continuación, fueron postfijadas en tetróxido de osmio al $2 \%$ en el mismo tampón durante una hora y se procedió a la deshidratación de las mismas, utilizando una serie de etanoles de gradación creciente $\left(50^{\circ}, 70^{\circ}, 90^{\circ}, 95^{\circ} \mathrm{y}\right.$ absoluto), para proceder a su inclusión en una resina epoxy, Epón-812

Una vez polimerizados, los bloques fueron cortados en un ultramicrotomo, realizando cortes semifinos de $1 \mu \mathrm{m}$ de espesor, teñidos con azul de toluidina al 0,5\%, para su observación a microscopia óptica. Por su parte, cortes ultrafinos de $70 \mathrm{~nm}$ de grosor se contrastaron con acetato de uranilo (al 5\%) y citrato de plomo (al 2,5\%) para su observación al microscopio electrónico.

\section{Procesamiento de retinas para estudios con la técnica de TUNEL.}

A. Aplicación de la Técnica de TUNEL sobre retinas enteras. Para estos estudios, se utilizaron 3 retinas de cada uno de los siguientes estadios: S16, S18, S19, S20, S22, S24 y S26. Las retinas fueron procesadas mediante la técnica TUNEL descrita por Gavrielli et al (1992), usando el kit de detección celular in situ POD. Previamente a la tinción con esta técnica, las retinas fueron incubadas en $1 \%$ de tritón X-100 en PBS durante 45 minutos. Posteriormente fueron lavadas en PBS y tratadas con peroxidasa al 3\% durante 15 minutos para eliminar la peroxidasa endógena. A continuación se lavó en agua destilada. Seguidamente fueron incubadas con proteinasa $\mathrm{K}(20 \mu \mathrm{m} / \mathrm{ml})$ en Tris/ $\mathrm{HCl}$ durante 20 minutos y lavadas 3 veces en PBS durante 15 minutos. Las retinas fueron incubadas durante una hora a $37^{\circ} \mathrm{C}$ con la reacción mixta TUNEL. A continuación, se lavaron en PBS y se cubrieron 30 minutos con POD a $37^{\circ} \mathrm{C}$. Posteriormente, se lavaron en tampón Tris (TB), y la actividad peroxidasa fue revelada con $0,05 \%$ de diaminobencidina (DAB) durante 4-5 min. Las retinas fueron lavadas en TB y después en $\mathrm{PB}$, y se extendieron sobre un porta con el lado vítreo hacia arriba con glicerol.

B. Distribución de las células TUNEL-positivas en retinas enteras. Una vez realizada la técnica de TUNEL, las imágenes con las células marcadas fueron importadas a un ordenador para su análisis morfométrico.

B. 1. Topografía y densidad. Para determinar la densidad de células TUNEL+ de las zonas central y periférica, de cada retina, se utilizaron tres retinas enteras de cada uno de los estadios estudiados. En todas las áreas de las muestras se han visto células TUNEL+, a diferentes profundidades focales correspondientes a la GCL y la INL respectivamente (la ONL no se tuvo en cuenta porque la presencia de células TUNEL+ era muy escasa). Tras los recuentos, se estimaron las densidades medias de células TUNEL+, tanto en la GCL como en la INL de toda la retina. En los resultados no se ha tenido en cuenta el factor de retracción del tejido durante la realización de la técnica, debido a que éste era insignificante.

B. 2. Análisis espacial de los datos. Para conocer el patrón de distribución de nuestra población celular se utilizó la aplicación informática, Spattial Patterns 2.3.2, un programa BASIC escrito usando Microsoft QuickBasic compilado para Apple Macintosh (Fernández, 1996). El programa permite la colección y el análisis de datos que pueden ser introduci- 
dos a partir de una tabla digitalizada o directamente importando coordenadas $\mathrm{x}, \mathrm{y}$ de diferentes procesadores, como por ejemplo Word, a programas de análisis de imagen. Además, el programa proporciona de manera rápida, fácil e interactiva el acceso a las técnicas estadísticas, matemáticas y gráficas usadas en el análisis. Esas técnicas incluyen varias medidas de dispersión (contar cuadrados, el cálculo del vecino más próximo y un análisis bidimensional de autocorrelación de puntos) y medidas de distribución.

Procesamiento de las retinas para estudios de inmunocitoquímica. Para la detección de las ribonucleoproteínas se utilizó una técnica que detecta específicamente RNA sobre cortes ultrafinos tanto de EPON como de LRWhite (Calabuig et al. 2005). Esta técnica se realizó en retinas de embriones de tortuga del estadio S24 incluidas en Epón-812.

Brominación. Primeramente, se procedió a la incubación de las rejillas de níquel, donde se encontraba el tejido, con bromo durante $30 \mathrm{~min}$ (Bromo $0,025 \%$ en bromuro de potasio $0,2 \%$ ). Posteriormente se hicieron 5 lavados de 5 minutos cada uno con agua destilada y se procedió al lavado de las muestras con bisulfito sódico al 0,5\%. Inmediatamente se volvieron a lavar en agua destilada (4 veces durante 5 minutos cada vez) y en PBS (4 veces durante 10 minutos cada vez).

Inmunomarcado. Primero se procedió al bloqueo con una solución de PBS (pH 8) con 0,01\% Twen 20, 2\% BSA, $100 \mu \mathrm{m}$ Glicina, y $2 \%$ Fetal Calf FERUM durante 30 minutos. A continuación, las muestras se incubaron con el anticuerpo primario. En el caso de la BrU se utilizó "mouse anti-BrdU" a la concentración de $1 / 100$, durante $1 \mathrm{~h}$ a temperatura ambiente. Seguidamente, se hicieron 4 lavados en PBS. Como anticuerpo secundario se utilizó "Inmuno Gold Congudate Goat antimouse Ig G", a una concentración de 1/50 durante $1 \mathrm{~h}$. Finalmente se hicieron 5 lavados de PBS y de agua destilada.

Contraste de rejillas. Las rejillas fueron contrastadas con acetato de uranilo (al 5\%) y citrato de plomo (al 2,5\%) para su observación al microscopio electrónico.

\section{RESULTADOS}

Apoptosis durante el desarrollo embrionario de la retina de tortuga. Los primeros signos de apoptosis en la retina de tortuga comienzan en el estadio S16 en la INL. Sin embargo, no se manifiesta claramente, tanto en la INL como en la GCL, hasta S18. La presencia de células TUNEL+ en la ONL es muy escasa.

Estudios de microscopía óptica. En secciones verticales de retina, en especial del es-

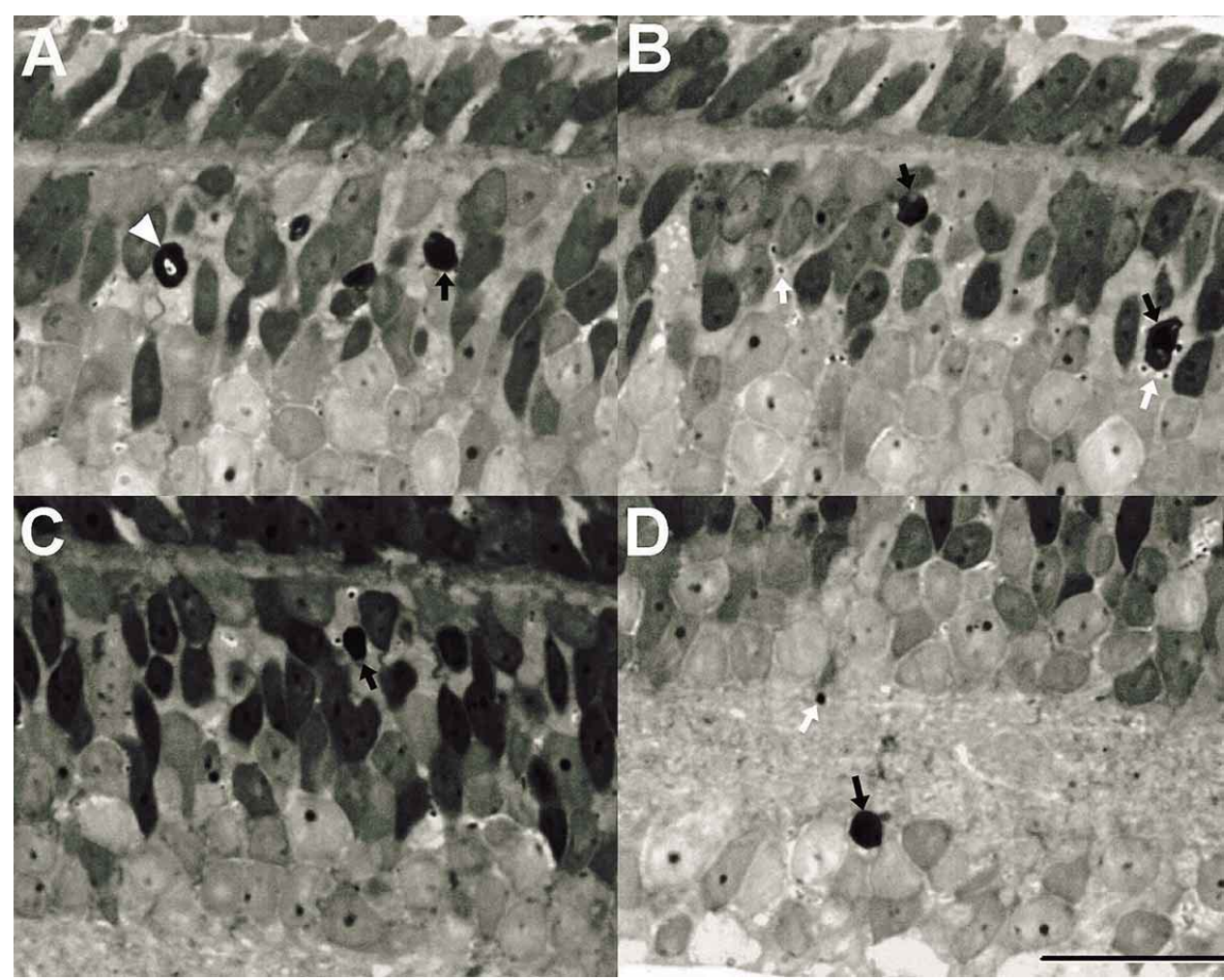

Fig. 1. Cortes semifinos de retinas de embriones de tortugas en el estadio S20. Barra $=25 \mu \mathrm{m}$. tadio S20, aparece un discreto número de células apoptóticas dispersas, caracterizadas por presentar un núcleo con pequeñas o grandes masas de cromatina condensada (Fig. 1). Algunos de estos núcleos presentan un aspecto picnótico debido a la distribución homogénea de heterocromatina por todo el carioplasma (Fig. 1, flechas negras). En otros casos, la cromatina se condensa en la periferia, por debajo de la envoltura nuclear, dándole al núcleo un típico aspecto anular (Fig. 1A, flecha blanca). También se observa que las células apoptóticas presentan fragmentos nucleares que se corresponden con los denominados cuerpos apoptóticos (Fig. 1B y 1D, flechas blancas). En general, la inmensa 
mayoría de las células apoptóticas han sido observadas en la GCL y en la INL.

Estudios de retinas enteras con el método TÚNEL. En retinas enteras extendidas se observan células apoptóticas desde el estadio S16 hasta el S24, y desaparecen en S26, tanto en la INL como en la GCL. Sin embargo, la mayor densidad de células TUNEL+ por $\mathrm{mm}^{2}$ ha sido observada entre S19 y S20, cuadruplicando los valores más bajos (Fig. 2). Por otra parte, la densidad de células TUNEL+, tanto en la INL como en la GCL, es mayor en áreas de la retina central respecto de la periférica, en particular entre los estadios S16 y S19. Entre S20 y S26 la densidad de las células TUNEL+ es menos dispar entre áreas de la retina central y periférica y no muestran diferencias significativas para una $\mathrm{p}<0,05$.

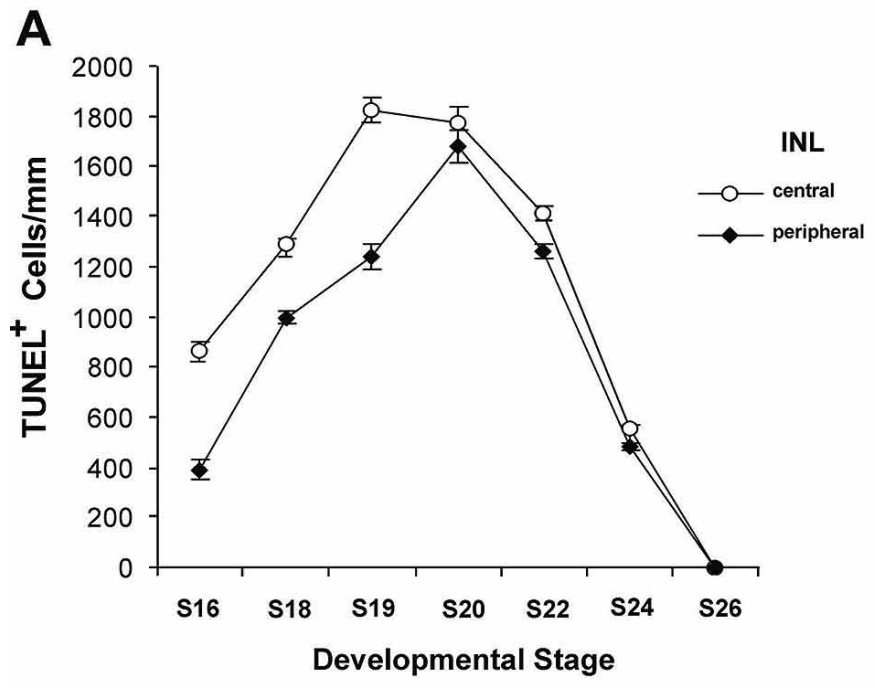

B

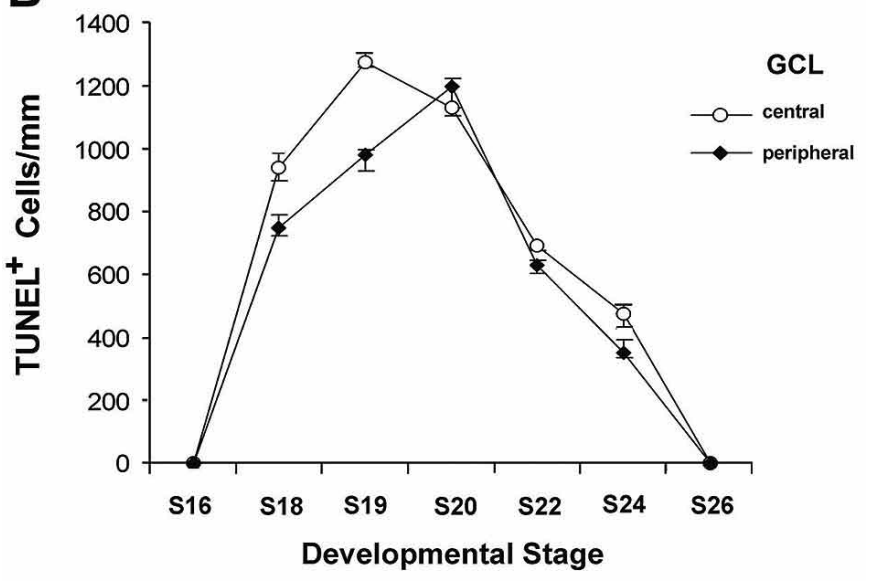

Fig. 2. Densidad de células marcadas positivamente con el método de TUNEL en la INL y en la GCL, tanto de la zona central como periférica de la retina.
Para determinar si la distribución de las células TUNEL+ es aleatoria o por el contrario sigue algún tipo de patrón espacial en retinas extendidas, se realizaron diversos análisis utilizando la aplicación informática, Spattial Patterns 2.3.2 (Fernández). Para este análisis se estudiaron las células apoptóticas de la INL y GCL de S20, por ser el estadio que presentó mayor densidad de células TUNEL+ (Ver Fig. 2). Aunque los datos obtenidos con los diferentes índices, ratios y test (Tabla I), proporcionaron resultados ambivalentes, del análisis de las células TUNEL+ podemos deducir que existe una marcada tendencia a distribuirse de una manera regular o agrupada. En efecto, los tres parámetros de dispersión, del método de los cuadrados (Dispersion index, Lefkovith's index y Quadrat count) utilizados para establecer el tipo de distribución de las células apoptóticas en la INL y GCL, tanto en la periferia como en la región central de la retina confirman ese tipo de distribución de las células TUNEL+, excepto en la INL de la retina central, que no sigue una distribución regular. Sin embargo, los parámetros analizados con el método de la distancia del vecino más próximo proporcionan resultados ambivalentes.

Estudios de microscopía electrónica. En micrografías electrónicas, el número de células apoptóticas se presentan con mayor densidad en S20, como hemos observado en los estudios de microscopia óptica. En general, las células apoptóticas son muy difíciles de observar en todos los estadios, excepto en S20, donde los perfiles apoptóticos son mucho más frecuentes. A nivel ultraestructural, los núcleos de las células apoptóticas manifiestan importantes cambios de su arquitectura. Esos cambios afectan tanto a la cromatina como a la apariencia de estructuras relacionadas con las ribonucleoproteínas (RNP) contenidas en el núcleo y en el nucléolo.

A. Cambios ultraestructurales durante la degradación de la cromatina en la apoptosis. Las micrografías electrónicas muestran diferentes señales relacionadas con las células apoptóticas de la retina. En el estadio S20 con frecuencia hay presencia de diferentes patrones de condensación de la cromatina nuclear tanto en la INL como en la GCL. Los neuroblastos apoptóticos tempranos muestran condensación y marginación de la cromatina (Fig. 3A) y una envoltura nuclear bien conservada. Tan pronto como la apoptosis progresa, observamos diferentes patrones nucleares de condensación de la cromatina: desde un núcleo en el cual la cromatina condensada se localiza en la periferia nuclear, debajo de la carioteca, con aspecto de anillo (Fig. 3B), hasta un núcleo cuya cromatina homogénea y condensada ocupa todo el interior nuclear, dando un aspecto picnótico (Fig. 3F), pasando por núcleos con aspecto de "media luna" (Fig. 3E). 


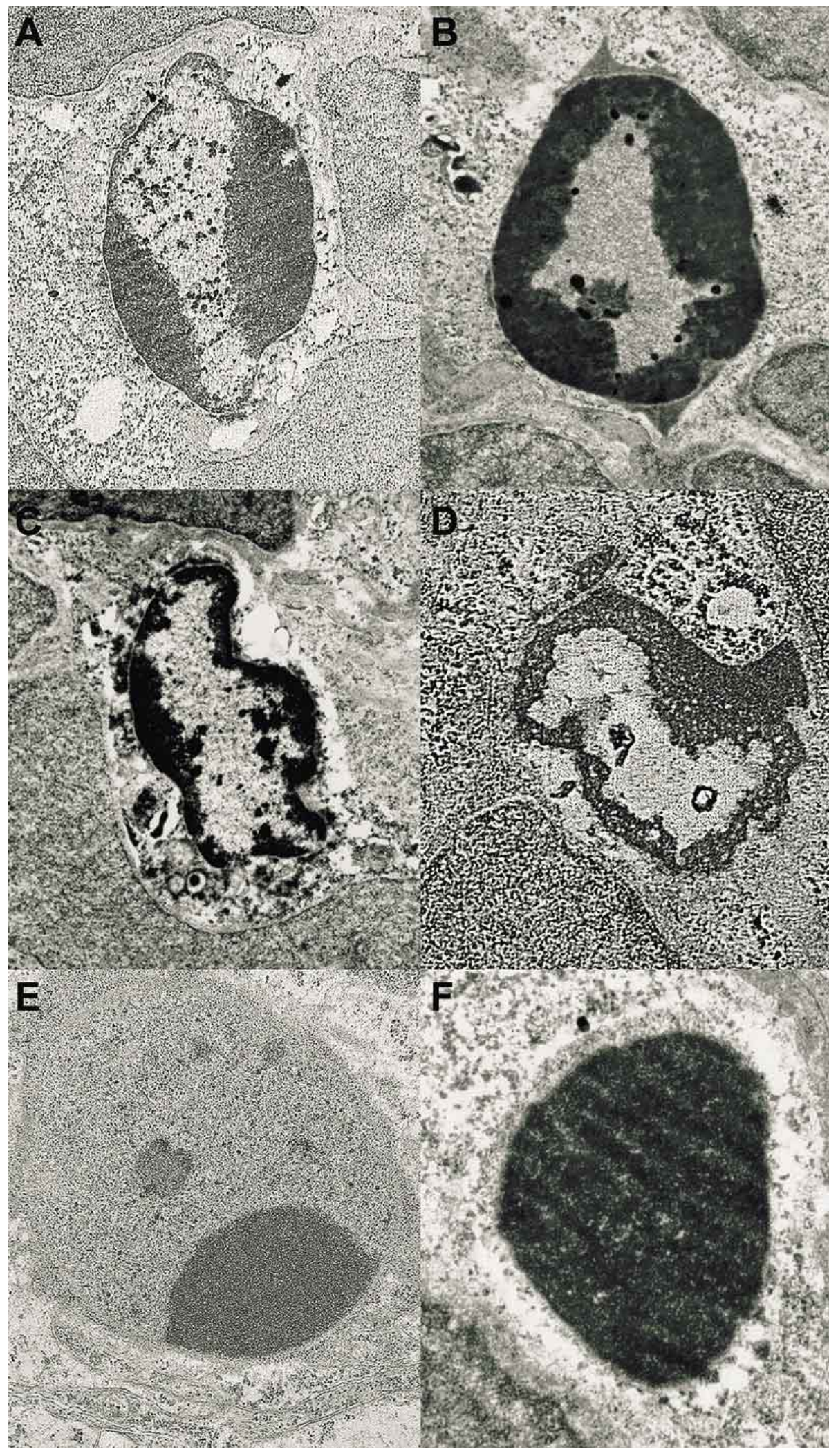

Fig. 3. Micrografía electrónica de diferentes patrones de distribución de la cromatina condensada en células apoptóticas de la retina de embriones de tortuga.
En estadios más avanzados de apoptosis, los neuroblastos experimentan rotura de la carioteca, cariorrexis y fragmentación en múltiples cuerpos apoptóticos (Fig. 4) que ocupan el citoplasma de las células con ruptura de la membrana plasmática y de las organelas.

\section{B. Cambios ultraestructurales} en las ribonucleoproteínas. En la retina de Trachemys scripta elegans se observan otros cambios ultraestructurales relacionados con la muerte celular y con estructuras que contienen las RNP. En este sentido, los primeros cambios ultraestructurales observados fueron la presencia de una desorganización del nucléolo (Fig. 5), caracterizada por la homogeneidad de su material, lo que le daba un aspecto amorfo. Estos cambios se observan especialmente en retinas de S18 y S20. En estos estadios además, es frecuente la presencia de cambios degenerativos en el citoplasma (Fig. 3). Finalmente, un hallazgo característico de este trabajo es la presencia de masas amorfas de material más o menos electrodenso disperso, tanto en el carioplasma como en el citoplasma (Fig. 6). Ese material se agrupa en acúmulos que han recibido el nombre de HERDS (Heterogeneous ectopic ribonucleoprotein-derived structures) por Biggiogera et al. (2004).

Utilizando la técnica de Calabuig et al. (2005), hemos podido demostrar, con microscopía electrónica, la presencia de RNA en los HERDS. Con esta técnica, el RNA aparece marcado como clusters de partículas de oro coloidal (10 $\mathrm{nm}$ ) ubicados sobre las masas de HERDS (Fig. 7). 

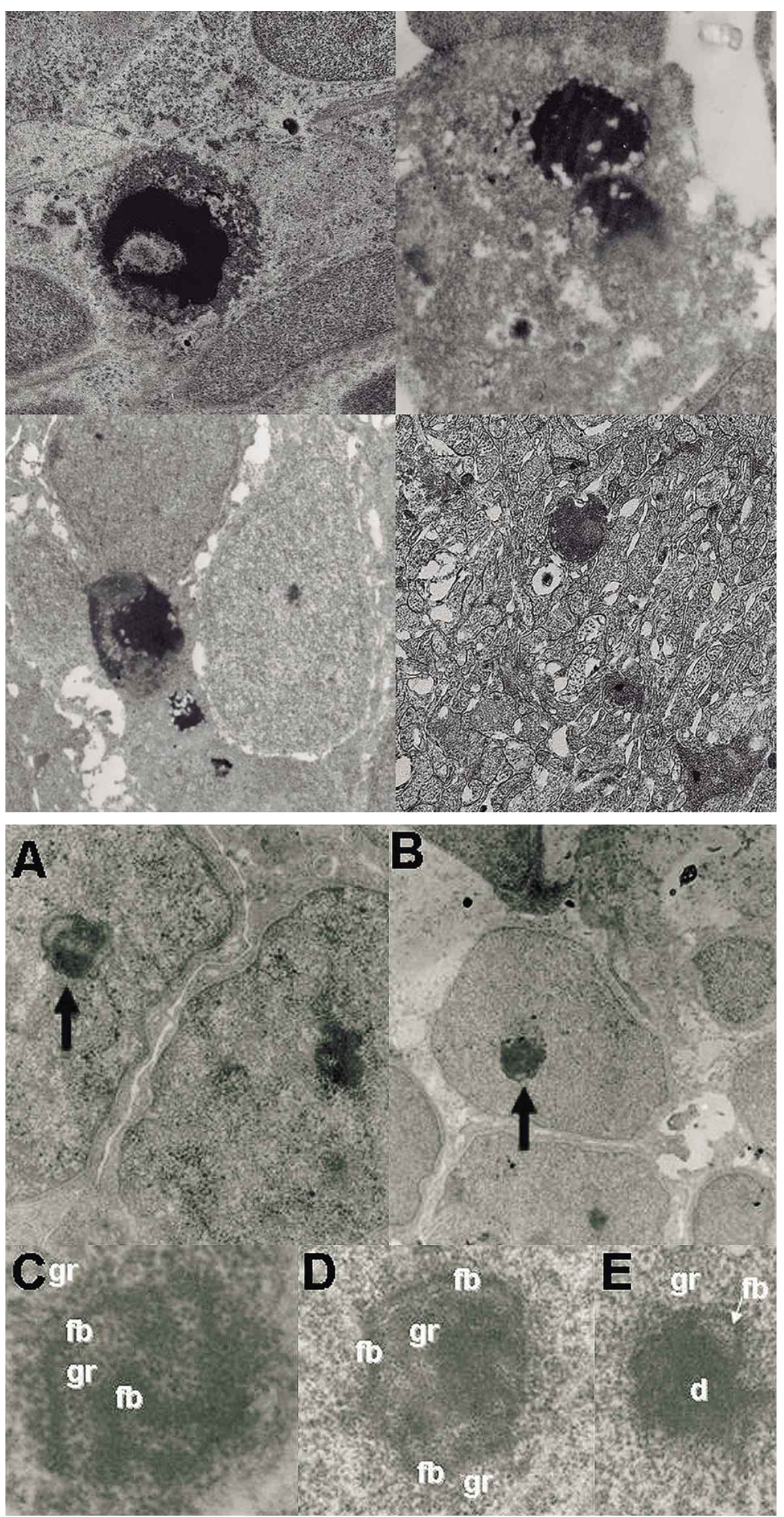

Fig. 4. Micrografía electrónica de diferentes patrones de distribución de la cromatina condensada de diferentes cuerpos apoptóticos.

Fig. 5. Micrografía electrónica de diferentes patrones de distribución de los diferentes componentes de los nucléolos tales como la pars granulosa (gr), pars fibrosa (fb) y pars densa (d). Obsérvese la tendencia a disgregarse el nucléolo y la tendencia a predominar la pars densa. 


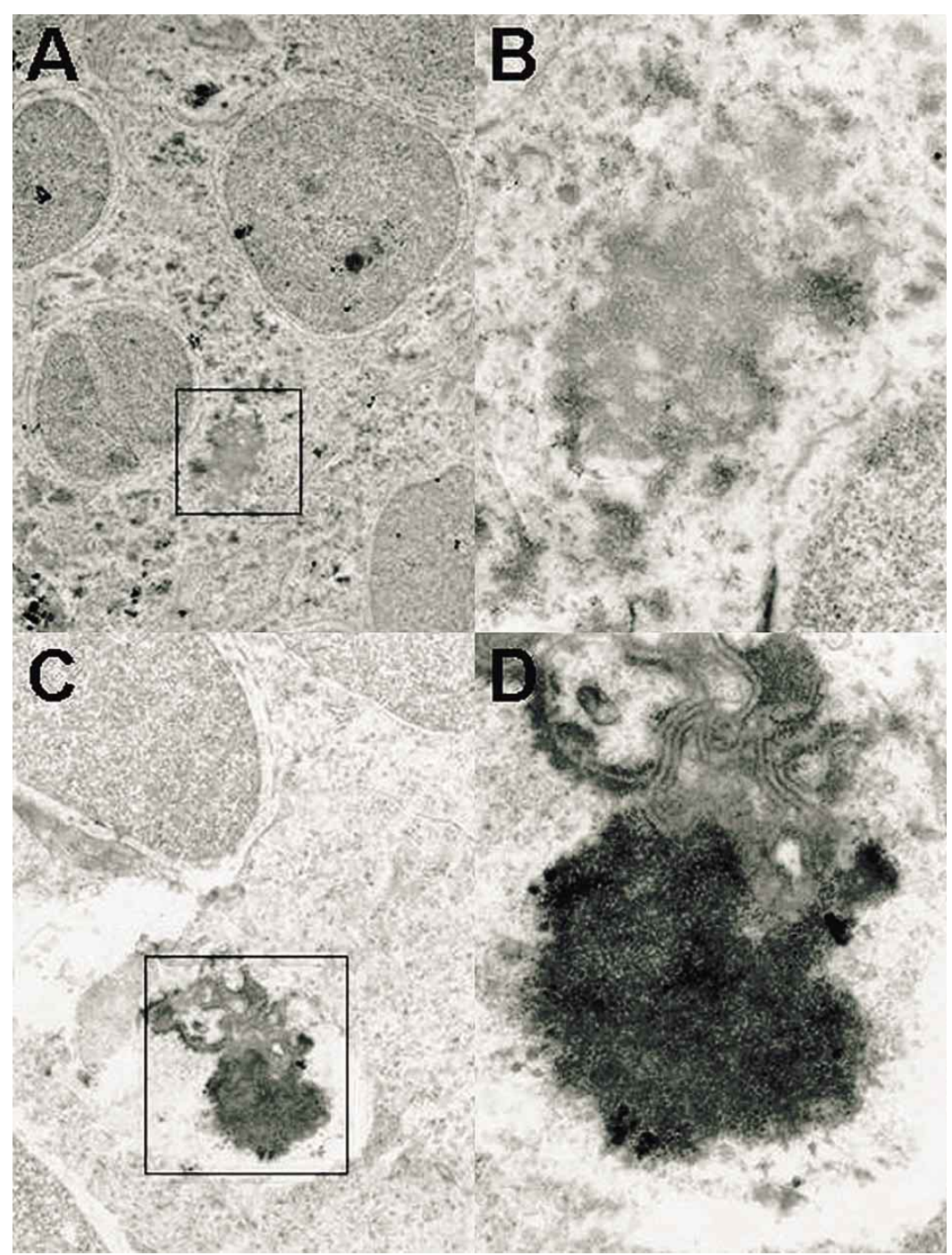

Fig. 6. Micrografía electrónica de diferentes formas de manifestarse el material ribonucleoproteinico extruido por el núcleo al citoplasma (HERDS) en la apoptosis.

Fig. 7. Micrografía electrónica de HERDS marcados con oro coloidal que indican la presencia de RNA.

\section{DISCUSIÓN}

Características morfológicas y distribución de la apoptosis. La apoptosis se encuentra ampliamente documentada en el desarrollo del sistema nervioso en general (Buss et al., 2006; Valenciano et al., 2009) y en el sistema visual en particular (Cellerino et al., 2000; Bejarano-Escobar et al., 2013). Como se describe en los resultados, los primeros signos de apoptosis en la retina de Trachemys scripta elegans comienzan en S16 en la INL, alcanzan su máxima densidad, tanto en la INL como en la GCL en S20 y se extinguen en S26, siguiendo un patrón muy similar al descrito por Francisco-Morcillo et al. (2004) en la retina de Mauremys leprosa. Las características morfológicas en microscopía óptica son similares a las observadas en la retina de otra especie de tortuga, Mauremys leprosa (Francisco-Morcillo et al.), así como de otros vertebrados, como peces (Hoke \& Fernald, 1998; Cole \& Ross, 2001), anfibios (Gaze \& Grant, 1992) aves (Martín-Partido et al., 1988; Marín-Teva et al.; Díaz et al., 2000), mamíferos inferiores, como la rata (Young; Galli-Resta \& Ensini, 1996; Cusato et al., 2001) y humanos (Marín-Teva et al.). Estas características pueden resumirse en la presencia de pequeñas o grandes masas de cromatina condensada en el núcleo, núcleos con aspecto picnótico debido a la distribución homogénea de la heterocromatina por todo el carioplasma o en la periferia, por debajo de la envoltura nuclear, así como en forma de cuerpos apoptóticos. En general, la inmensa mayoría de las células apoptóticas han sido observadas en la GCL y en la INL siendo difícil de ver en la ONL (MarínTeva et al.; Francisco-Morcillo et al.).

Respecto a la cronología en la aparición y evolución de la apoptosis, observada con el método TUNEL, nuestros resultados son superponibles a los de Francisco-Morcillo et al. En efecto la apoptosis se inicia alrededor de S16, presentando su máxima expresión alrededor de S20, para extinguirse en S26. Este patrón es prácticamente similar en la INL y en la GCL, con 


\begin{tabular}{|c|c|c|c|c|}
\hline Stage & \multicolumn{2}{|c|}{$\begin{array}{c}\mathrm{S} 20 \\
\text { Inner Nuclear Layer }\end{array}$} & \multicolumn{2}{|c|}{$\begin{array}{c}\mathrm{S} 20 \\
\text { Ganglion Cell Layer }\end{array}$} \\
\hline Parameters & Central & Periphery & Central & Periphery \\
\hline $\begin{array}{l}\text { Number of cells } \\
\text { (n) }\end{array}$ & (130) & $(52)$ & (71) & (45) \\
\hline \multicolumn{5}{|c|}{ Quadrat method } \\
\hline $\begin{array}{l}\text { Frequency } \\
\text { (mean by tile + SD) }\end{array}$ & $\begin{array}{l}3.556 \pm \\
1.832\end{array}$ & $\begin{array}{l}1.417 \pm \\
1.498\end{array}$ & $\begin{array}{l}1.917 \pm \\
1.552\end{array}$ & $\begin{array}{l}1.194 \pm \\
1.430\end{array}$ \\
\hline Dispersion index & $\begin{array}{c}0.944 \\
\text { RA/RE }\end{array}$ & $\begin{array}{l}1.583 \\
\text { cluster }\end{array}$ & $\begin{array}{l}1.257 \\
\text { cluster }\end{array}$ & $\begin{array}{l}1.713 \\
\text { cluster }\end{array}$ \\
\hline Lefkovithus index & $\begin{array}{l}-0.036 \\
\text { RA/RE }\end{array}$ & $\begin{array}{l}0.283 \\
\text { cluster }\end{array}$ & $\begin{array}{l}0.144 \\
\text { R/cluster }\end{array}$ & $\begin{array}{c}0.327 \\
\text { RA/cluster }\end{array}$ \\
\hline $\begin{array}{l}\text { Quadrat count } \\
\text { T statistic }\end{array}$ & $\begin{aligned} p= & 0.790 \\
t= & 0.826 \\
& R A\end{aligned}$ & $\begin{array}{l}p=0.001 \\
t=3.457 \\
\text { cluster }\end{array}$ & $\begin{array}{l}p=0.022 \\
t=2.063 \\
\text { cluster }\end{array}$ & $\begin{array}{l}p=0.001 \\
t=3.560 \\
\text { cluster }\end{array}$ \\
\hline \multicolumn{5}{|c|}{ Nearest Neighbor Distance (NND) analysis } \\
\hline $\begin{array}{c}\text { NND }(\mu \mathrm{m}) \\
(\text { mean } \pm S D, n)\end{array}$ & $\begin{array}{c}23.50 \pm 10.68 \\
(130)\end{array}$ & $\begin{array}{c}31.64 \pm 22.32 \\
(52)\end{array}$ & $\begin{array}{c}26.37 \pm 21.59 \\
(71)\end{array}$ & $\begin{array}{c}35.92 \pm 17.43 \\
(45)\end{array}$ \\
\hline $\begin{array}{l}\text { Chi normal } \\
\text { Chi Raleigh }\end{array}$ & $\begin{array}{c}p=0.001 \\
\text { no normal } \\
p=0.001 \\
\text { No RA }\end{array}$ & $\begin{array}{c}p=0.520 \\
\text { normal } \\
p=0.992 \\
R A\end{array}$ & $\begin{array}{c}p=0.002 \\
\text { no normal } \\
p=0.134 \\
\text { RA }\end{array}$ & $\begin{array}{c}p=0.210 \\
\text { normal } \\
p=0.448 \\
\text { RA }\end{array}$ \\
\hline Clark \& Evans test & $p=0.776$ & $\begin{array}{c}p=0.138 \\
R A\end{array}$ & $\begin{array}{l}p=0.014 \\
\text { cluster }\end{array}$ & $\begin{array}{c}p=0.812 \\
R A\end{array}$ \\
\hline $\begin{array}{l}\text { Conformity ratio } \\
\text { (mean/SD of NND) }\end{array}$ & $\begin{array}{l}2.20 \\
\text { RE }\end{array}$ & $\begin{array}{l}1.42 \\
\text { RE }\end{array}$ & $\begin{array}{l}1.22 \\
\mathrm{RE}\end{array}$ & $\begin{array}{l}2.06 \\
\text { RE }\end{array}$ \\
\hline $\begin{array}{l}\text { Effective ratio } \\
\text { ( } \mu \mathrm{m} ; \text { from DRP) }\end{array}$ & $\begin{array}{l}9.19 \\
7.93\end{array}$ & $\begin{array}{c}9.86 \\
10.00\end{array}$ & $\begin{array}{l}8.89 \\
8.32\end{array}$ & $\overline{--}$ \\
\hline Eberdhart index & $\begin{array}{c}1.21 \\
\text { RA/RE }\end{array}$ & $\begin{array}{c}1.50 \\
\text { cluster }\end{array}$ & $\begin{array}{c}1.67 \\
\text { cluster }\end{array}$ & $\begin{array}{c}1.24 \\
\text { RA/RE }\end{array}$ \\
\hline Equilateral index & $\begin{array}{c}0.645 \\
\text { RA }\end{array}$ & $\begin{array}{c}0.695 \\
\text { RA }\end{array}$ & $\begin{array}{c}0.677 \\
\text { RA }\end{array}$ & $\begin{array}{c}0.664 \\
\text { RA }\end{array}$ \\
\hline RNNA & $\begin{array}{c}p=0.28 \\
R A\end{array}$ & $\begin{array}{c}p=0.31 \\
R A\end{array}$ & $\begin{array}{l}p=0.01 \\
\text { cluster }\end{array}$ & $\begin{array}{c}p=0.27 \\
R A\end{array}$ \\
\hline
\end{tabular}

Tabla I. Resultados de los diferentes análisis realizados con la aplicación Spattial Pattern 2.3.2. para determinar los patrones de agrupación de las células TUNEL+ en S20. 
tan solo diferencias en la intensidad del proceso. Otra similitud entre la apoptosis de Mauremys leprosa (FranciscoMorcillo et al.) y Trachemys scripta elegans, es la evolución de la apoptosis desde el centro de la retina hacia la periferia, fenómeno que también se observa en las retinas de los vertebrados arriba mencionados.

Sin embargo, existen tres características diferenciales muy llamativas entre nuestros resultados y los de Francisco-Morcillo et al. En primer lugar la densidad de núcleos apoptóticos de la INL, en el estadio de máxima densidad (S20), es cuatro veces mayor en Trachemys scripta elegans que en Mauremys leprosa (Francisco-Morcillo et al.). De igual manera, la densidad en la GCL es 12 veces mayor en nuestro material que en el de Francisco-Morcillo et al. y finalmente, mientras que en la INL de Trachemys scripta elegans hay una densidad de núcleos apoptóticos 1,5 veces mayor que en la GCL, en Mauremys leprosa la densidad es 4 veces mayor en la INL que en la GCL. La mayor densidad de núcleos apoptóticos en Trachemys scripta elegans, puede ser debido a varias razones. La primera a considerar seria que realmente exista una diferencia entre ambas especies, en cuanto al número de sus células normales y/o apoptóticas. Consideramos esto poco probable dada la cercanía taxonómica de ambas especies y su mismo hábitat. Otra posibilidad sería un marcaje diferente con la técnica de TUNEL, debido al distinto procesamiento de las retinas. $\mathrm{La}$ técnica de TUNEL, en nuestro material, fue realizada sobre retinas enteras extendidas mientras que en el trabajo de Francisco-Morcillo et al. la técnica se aplicó sobre cortes de retinas incluidas en parafina.

En cuanto a la mayor densidad de núcleos TUNEL+ en la INL, respecto de la GCL, se explica fácilmente por la mayor densidad de células en esta capa respecto de las otras capas celulares (Francisco-Morcillo et al.). Dos son las hipótesis manejadas para la interpretación de esta marcada apoptosis. La primera explicaría la apoptosis de la INL como un fenómeno dependiente de las dianas. Esta hipótesis está avalada por el hecho de que la ablación del tectum óptico provoca un incremento de la apoptosis tanto en la GCL como en la INL, es decir, al disminuir los contactos de las neuronas con sus respectivas dianas. Sin embargo existe otra hipótesis alternativa por la que la apoptosis seria un fenómeno independiente de las dianas. En varios estudios realizados en pollo (Hering \& Kröger, 1996), codorniz (Marín-Teva et al.), ratón (Young) y rata (Horsburgh \& Sefton) se observa que la apoptosis de la INL precede a la aparición de sinapsis en la IPL. En la retina de tortuga, la aparición de las primeras sinapsis en la IPL se produce en el estadio S20 aunque de una manera muy escasa, y no es hasta S22 que se muestran claramente (Segovia et al., 2012).
Un resultado importante de este trabajo fue comprobar que existe una marcada tendencia de las células TUNEL+ a distribuirse de una manera regular o agrupada (Ver Tabla I). En general encontramos campos en los que se observa una clara distribución en grupos o de forma regular, frente a otros en los que la distribución es claramente aleatoria. Existen datos en la literatura que señalan que una distribución no aleatoria de las neuronas retinianas sería necesaria para la formación de patrones de conectividad durante el desarrollo (Cameron \& Carney, 2004). Esto es importante ya que la formación de patrones celulares de distribución en la retina es un fenómeno extremadamente complejo. Un primer requerimiento para el normal funcionamiento de la retina es que los millones de células que la componen, pertenecientes a más o menos una decena de tipos neuronales (fotorreceptores, bipolares, ganglionares, horizontales, amacrinas e interplexiformes) además de la glía, deben estar adecuadamente dispuestas en un espacio tridimensional.

Respecto a la microscopía electrónica, las células apoptóticas son muy difíciles de observar en la mayoría de los estadios y algo más fácil en S20. A nivel ultraestructural, los núcleos de las células apoptóticas manifiestan importantes cambios de su estructura. Los cambios observados en este trabajo son superponibles a los observados, por otros autores, en la cromatina de otras células (Rogalinska, 2002). También se observan cambios en las ribonucleoproteínas (RNP) del nucléolo, fenómeno descrito en otras localizaciones por Biggiogera et al.

En conclusión, la retina de Trachemys scripta elegans presenta las siguientes características acerca del proceso de apoptosis:

- Los fenómenos de apoptosis observados se producen fundamentalmente en la INL y en la GCL, en este orden, de mayor a menor intensidad, siendo más intensa en la retina central que en la periférica.

- La apoptosis parece ser un fenómeno independiente de las dianas puesto que precede a la aparición de las sinapsis en la IPL. Nuestros datos sugieren que podría funcionar como un mecanismo de remodelado neuronal para facilitar el crecimiento de las prolongaciones neuronales que establecerán los subsiguientes contactos sinápticos.

- Las células apoptóticas tienden a adoptar un patrón de distribución más o menos agrupado o no aleatorio.

- La apoptosis de las células de la retina embrionaria de tortuga presenta características microscópicas y ultraestructurales análogas a las de otras localizaciones del Sistema Nervioso 
Central, a saber: a) condensación de la cromatina nuclear según diferentes patrones, b) formación de cuerpos apoptóticos fagocitados por las células vecinas y c) extrusión de ribonucleoproteínas al carioplasma y al citoplasma de la célula apoptótica. En este estudio hemos verificado además la presencia de RNA en la composición de estas estructuras.

SEGOVIA, Y. Apoptosis in turtle embryonic retina (Trachemys scripta elegans). Int. J. Morphol., 33(1):119-129, 2015.

SUMMARY: Apoptosis or programmed cell death is a process that occurs during development of the nervous system. The aim of this study was to observe the patterns of apoptosis that occur during embryonic development of the retina from the stage S16 at birth, by light and electron miscoscopia. Turtle embryonic retinas were used for the study. Our data show that the first signs of apoptosis begins at stage S16 in the inner nuclear layer and reaches maximum density both in the inner nuclear layer and the ganglion cell layer in S20 until they practically disappear at the time of birth. Furthermore, apoptosis follows a gradient center-periphery.

KEY WORDS: Trachemys scripta elegans; Apoptosis; Retina; Turtle.

\section{REFERENCIAS BIBLIOGRÁFICAS}

Bejarano-Escobar, R.; Blasco, M.; Durán, A. C.; Martín-Partido, G. \& Francisco-Morcillo, J. Chronotopographical distribution patterns of cell death and of lectin-positive macrophages/ microglial cells during the visual system ontogeny of the smallspotted catshark Scyliorhinus canicula. J. Anat., 223(2):17184, 2013.

Biggiogera, M.; Bottone, M. G.; Scovassi, A. I.; Soldani, C.; Vecchio, L. \& Pellicciari, C. Rearrangement of nuclear ribonucleoprotein (RNP)-containing structures during apoptosis and transcriptional arrest. Biol. Cell, 96(8):603-15, 2004.

Buss, R. R; Sun, W. \& Oppenheim, R. W. Adaptive roles of programmed cell death during nervous system development. Annu. Rev. Neurosci., 29:1-35, 2006.

Calabuig, J.; de Juan, J. \& Iborra, F. J. Specific detection of RNA on ultra-thin sections. J. Struct. Biol., 152(2):146-8, 2005.

Cameron, D. A. \& Carney, L. H. Cellular patterns in the inner retina of adult zebrafish: quantitative analyses and a computational model of their formation. J. Comp. Neurol., 471(1):11-25, 2004.

Cellerino, A.; Bähr, M. \& Isenmann, S. Apoptosis in the developing visual system. Cell Tissue Res., 301(1):53-69. 2000.

Cole, L. K. \& Ross, L. S. Apoptosis in the developing zebrafish embryo. Dev. Biol., 240(1):123-42, 2001.

Cusato, K.; Stagg, S. B. \& Reese, B. E. Two phases of increased cell death in the inner retina following early elimination of the ganglion cell population. J. Comp. Neurol., 439(4):440-9, 2001.

Díaz, B.; Serna, J.; De Pablo, F. \& de la Rosa, E. J. In vivo regulation of cell death by embryonic (pro)insulin and the insulin receptor during early retinal neurogenesis. Development, 127(8):1641-9, 2000.
Fernández, E. Spatial Patterns. Versión 2.3.2. [Programa de ordenador]. Alicante, Instituto de Neurociencias, Universidad de Alicante, 1996.

Francisco-Morcillo, J.; Hidalgo-Sánchez, M. \& Martín-Partido, G. Spatial and temporal patterns of apoptosis during differentiation of the retina in the turtle. Anat. Embryol. (Berl.), 208(4):289-99, 2004.

Galli-Resta, L. \& Ensini, M. An intrinsic time limit between genesis and death of individual neurons in the developing retinal ganglion cell layer. J. Neurosci., 16(7):2318-24, 1996.

Gaze, R. M. \& Grant, P. Spatio-temporal patterns of retinal ganglion cell death during Xenopus development. J. Comp. Neurol., 315(3):264-74, 1992.

Glücksmann, A. Cell deaths in normal vertebrate ontogeny. Biol. Rev. Camb. Philos. Soc., 26(1):59-86, 1951.

Hering, H. \& Kröger, S. Formation of synaptic specializations in the inner plexiform layer of the developing chick retina. $J$. Comp. Neurol., 375(3):393-405, 1996.

Hoke, K. L. \& Fernald, R. D. Cell death precedes rod neurogenesis in embryonic teleost retinal development. Dev. Brain Res., 111(1):143-6, 1998.

Horsburgh, G. M. \& Sefton, A. J. Cellular degeneration and synaptogenesis in the developing retina of the rat. J. Comp. Neurol., 263(4):553-66, 1987.

Marín-Teva, J. L.; Cuadros, M. A.; Calvente, R.; Almendros, A. \& Navascués, J. Naturally occurring cell death and migration of microglial precursors in the quail retina during normal development. J. Comp. Neurol., 412(2):255-75, 1999. 
Martín-Partido, G.; Rodríguez-Gallardo, L.; Álvarez, I. S. \& Navascués, J. Cell death in the ventral region of the neural retina during the early development of the chick embryo eye. Anat. Rec., 222(3):272-81,1988.

Oppenheim, R. W. Cell death during development of the nervous system. Annu. Rev. Neurosci., 14:453-501, 1991.

Rogalinska, M. Alterations in cell nuclei during apoptosis. Cell Mol. Biol. Lett., 7(4):995-1018, 2002.

Segovia, Y.; Perez, R. M.; Grzywacz, N. M. \& De Juan, J. Does Müller Cell Differentiation Occur Prior to the Emergence of Synapses in Embryonic Turtle Retina? J. Life Sci. (Libertyville), 2012(6):1200-5, 2012.

Valenciano, A. I; Boya, P. \& de la Rosa, E. J. Early neural cell death: numbers and cues from the developing neuroretina. Int. J. Dev. Biol., 53(8-10):1515-28, 2009.

Yntema, C. L. A series of stages in the embryonic development of Chelydra serpentina. J. Morphol., 125(2):219-51, 1968.

Young, R. W. Cell death during differentiation of the retina in the mouse. J. Comp. Neurol., 229(3):362-73, 1984.
Correspondence to:

Dra. Yolanda Segovia

Dpto. Biotecnologia

Facultad de Ciencias

Universidad de Alicante

Alicante

ESPAÑA

Email: yolanda.segovia@ua.es

Recibido: 20-10-2014

Aceptado: 02-01-2015 